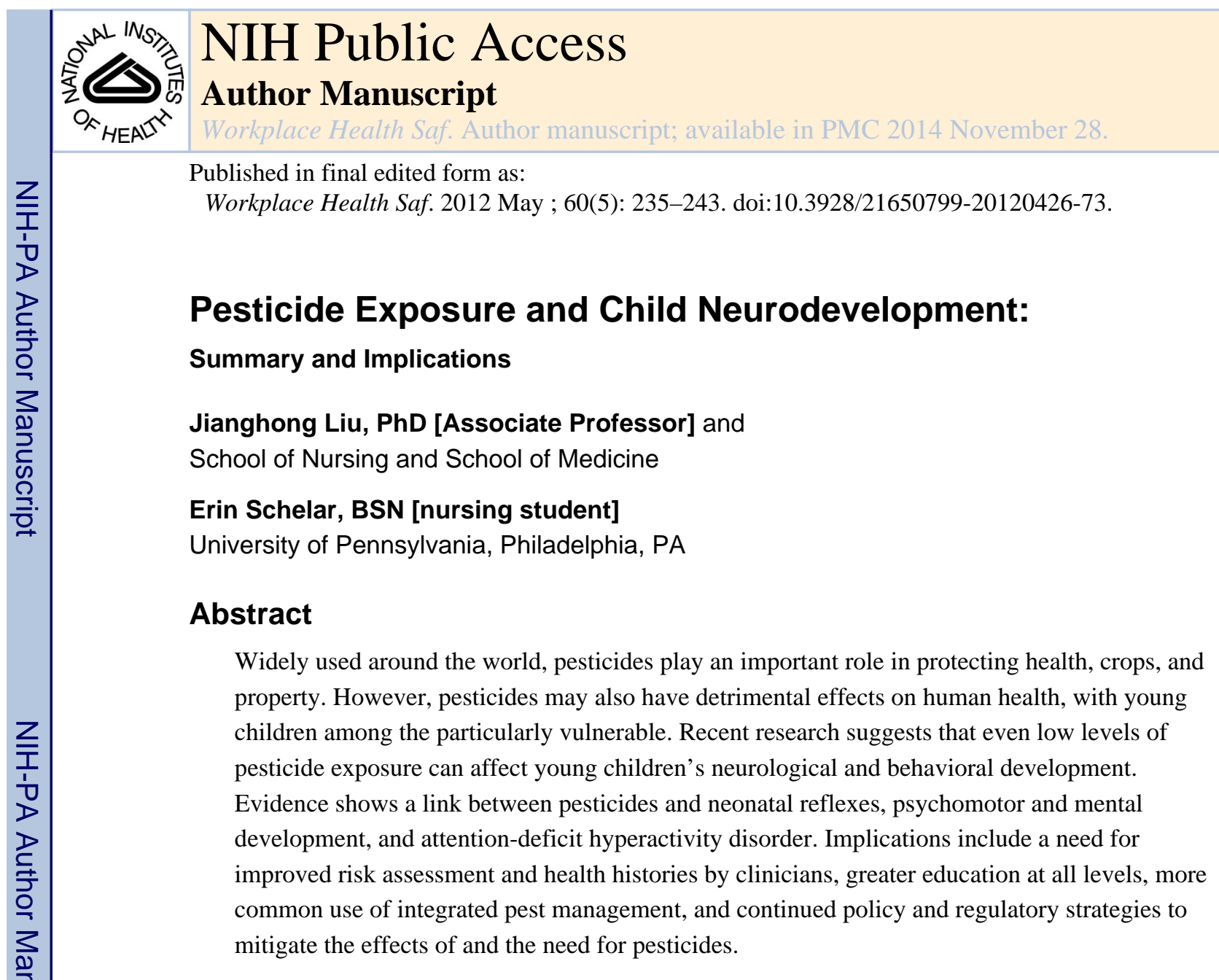

Pesticides are widely used both in the United States and around the world. The U.S. Environmental Protection Agency (EPA) has estimated that nearly 75\% of American households use pesticides, frequently targeting cockroaches and ants, and in total more than 1 billion tons of pesticide products are used each year commercially and agriculturally (EPA, 2010a). Pesticides are used not only in developed countries, but also in developing countries where less regulation protects the public from certain pesticides with deleterious effects or that may be used at any level (Ecobichon, 2001).

Pesticides have an important role in agriculture and public health. Farmers use pesticides to protect their crops. Residentially, pesticides may be used to eliminate rodents or insects, as these animals carry or cause disease or damage property. Pesticides encompass a broad category of substances, including insecticides, herbicides, fungicides, rodenticides, and fumigants, aimed at preventing, destroying, or repelling pests (Chalupka \& Chalupka, 2010), including insects (e.g., cockroaches), rodents (e.g., mice), microorganisms (e.g., bacteria), or unwanted plants (EPA, 2010a). Pesticides can be classified by the type of organisms they target or by their composition (e.g., chemicals, biopesticides, antimicrobials, or pest control devices). This article focuses on the effects of chemical pesticides.

Copyright (C) American Association of Occupational Health Nurses, Inc.

Address correspondence to Jianghong Liu, PhD, School of Nursing and School of Medicine, University of Pennsylvania, 418 Curie Blvd., Room 426, Claire M. Fagin Hall, Philadelphia, PA 19104-6096.jhliu@ nursing.upenn.edu.

The authors disclose that they have no significant financial interests in any product or class of products discussed directly or indirectly in this activity. 
Many categories of chemical pesticides must be registered with the EPA, the most prevalent being organophosphates, organochlorines, pyrethroids, and carbamates. Organophosphates are one of the most frequently used categories of chemical pesticides. Their action targets the nervous system by altering the effect of an enzyme that regulates select neurotransmitters. Although organophosphates are frequently used as insecticides, they rarely persist in the environment (EPA, 2010a). Organochlorines, such as DDT, have been closely regulated or removed from the market because they do persist in the environment and have many negative environmental and health consequences (EPA, 2010a). Pyrethroids and carbamates also target the nervous system (EPA, 2010a). Because these toxins have been developed to affect living creatures, these chemicals may affect humans adversely as well.

\section{Significance of the problem}

Young children are particularly vulnerable to toxicants in the environment, including pesticides. The National Research Council's 1993 report on pesticides in the diets of infants and children recognized that children require a special approach to risk assessment due to their unique vulnerabilities to environmental toxicants, including pesticides (Landrigan, 2011; Landrigan \& Goldman, 2011; Landrigan, Kimmel, Correa, \& Eskenazi, 2004; Lucas \& Allen, 2009; Quiros-Alcala et al., 2011). Children's organs are not fully developed until later in life. They continually experience critical periods in development; adverse exposures can cause permanent damage, particularly in utero (Chalupka \& Chalupka, 2010).

Children's behaviors and ability to interact with their physical environment change during different stages of growth and development and can place them at greater risk of exposure: children may crawl on the floor, explore objects orally, and play with items they find in the environment (Landrigan et al., 2004). For their weight, children consume more food and drink than adults, increasing their possible dietary exposure; dietary exposure is compounded by children's immature livers and excretory systems, which may be unable to effectively remove pesticide metabolites (Landrigan et al., 2004). These metabolites may block the absorption of critical nutrients in children's diets, which further affects health outcomes. Potential routes of exposure to pesticides include in utero or through breast milk, via ingestion of food contaminated with pesticides, and household exposures via dermal contact (Chalupka \& Chalupka, 2010). Children live closer to the ground than adults, which may increase their exposure to pesticides sprayed or precipitated there (Paulson \& Barnett, 2010).

In 1996, the federal government passed the Food Quality Protection Act, which set standards for acceptable levels of pesticides in food to protect infants and children, erring on the side of caution if not enough information is available and encouraging additional research (Landrigan et al., 2004). Studies have established the link between pesticide poisonings and negative health consequences. However, the association between low-level pesticide exposures over time and neurodevelopmental and behavioral outcomes is less certain. This article attempts to address this gap by discussing how pesticide exposure, even at low or commonly reported levels, may impact children's neurological health and behavior and ways nurses can intervene. 


\section{Method}

A literature search in PubMed, CINAHL, and the Cochrane Library of systematic reviews used the key words pesticides (with limits set to English, human, and children) and children, neurologic, and behavior; this search was repeated without the limitation of human studies. Searches were also conducted with the key words education (with limits set to children, English, and humans), policy, and practice. References in the articles identified in the databases were also evaluated for inclusion. Because the authors were primarily interested in exploring the effects of pesticides in use today, selected articles were limited to the past 10 years and excluded studies regarding polychlorinated biphenyls (PCBs).

\section{Links between Neurobeha vior and Pesticides}

Recent research has examined the link between pesticide exposure and children's health outcomes. Pesticides have been shown to affect a variety of body systems, including reproductive, endocrine, immune, and respiratory (Gilden, Huffling, \& Sattler, 2010). However, as developmental disorders (e.g., autism) and behavioral conditions (e.g., attention-deficit hyperactivity disorder [ADHD]) (Xu et al., 2011) become more prevalent, it is increasingly important to assess possible links between environmental exposures and neurological or behavioral outcomes in children.

Newborns and infants have rapidly developing neurological pathways and attain several developmental milestones in the first years of life (Sattler \& Davis del, 2008), making children particularly vulnerable to the effects of pesticides. A study in New York City found a possible link between prenatal indoor pesticide exposure and abnormal neonatal reflexes, although the authors cautioned they were unable to accurately identify to which pesticides mothers were exposed (Engel et al., 2007). Researchers, identifying the critical prenatal window when children may be particularly vulnerable to neurobehavioral effects of pesticides, monitored 224 mothers' serum levels of DDE, a metabolite of DDT, during each trimester of pregnancy and discovered that levels during the first trimester were linked to reduced psychomotor development index scores—but not mental development index scores —during the first year of life (Torres-Sanchez et al., 2007). In contrast, in a longitudinal study of Latino farmworker families in California, no link was observed between prenatal or childhood exposure to organophosphates in the first 2 years of life and psychomotor development. However, the researchers did find a negative association between prenatal exposure and mental development and pervasive developmental disorder at 24 months of age (Eskenazi et al., 2007). Interestingly, childhood exposure was positively associated with these same two outcomes (Eskenazi et al., 2007). Furthermore, in a prospective birth cohort of 3,421 pregnant women in an agricultural region in Brittany, France, negative effects on cranial growth were reported among 36-month-old children of women who were exposed to pesticides during pregnancy (Petit et al., 2010). Another study in New York City also found prenatal exposure to piperonyl butoxide to be negatively associated with 36-month neurodevelopment (Horton et al., 2011).

Children of preschool age are expected to reach neurological developmental milestones (e.g., verbalization, ambulation), but research suggests that pesticides may interfere with 
some of these. One Spanish cohort study assessed this relationship using the McCarthy Scales of Children's Abilities, questionnaires on children's diet and parental sociodemographics, and measurements of DDT and its metabolite, DDE, through cord serum concentrations at birth (Ribas-Fito et al., 2006). The authors concluded that higher levels of DDT were associated with poorer outcomes, including lower verbal and memory scale scores, after controlling for diet and background characteristics, even though the use of the pesticide had been limited in the area for decades. Motor skills were reportedly unaffected, and girls demonstrated greater functional deficits in language skills than boys (Ribas-Fito et al., 2006).

In addition to background pesticide exposure in the environment, children living in agricultural areas may be at increased risk of exposure related to take-home exposure from their parents' employment and location of their home relative to production fields (Roberts et al., 2007; Rohlman et al., 2005). On comparisons of 48- to 71-month-old children living in an agricultural area with higher levels of organophosphates to cohorts living in nonagricultural areas, no statistically significant differences were found on most of the eight tests on neurobehavioral performance; however, the children in non-agricultural areas performed better than the children from agricultural areas on measures of reaction speed and latency (Rohlman et al., 2005). A prospective cohort study found that children of African American or Dominican mothers prenatally exposed to higher levels of chlorpyrifos exhibited poorer outcomes on the Child Behavior Checklist and Bayley Scales of Infant Development II compared to children with lower levels of exposure (Rauh et al., 2006), including greater deficits in motor, mental, and attention abilities. Furthermore, cognitive and psychomotor delays increased across all three assessment periods (i.e., 12, 24, and 36 months) (Rauh et al., 2006). Limited evidence suggests that pesticide exposure during pregnancy via maternal residential proximity to fields treated with organochlorines, especially during critical prenatal neurodevelopmental periods and with greater amounts of product, is associated with greater risk of the child later being diagnosed with an autism spectrum disorder (Roberts et al., 2007), representing an area for further investigation.

School-aged children may also be vulnerable to negative health consequences of pesticide exposure. Long-term sequelae of acute organophosphate pesticide poisoning in infancy include inhibitory motor control deficits when children reach school age (Kofman, Berger, Massarwa, Friedman, \& Jaffar, 2006). Children prenatally exposed to pesticides may also suffer effects into their school-aged years. Children born to mothers with occupational pesticide contact exhibited deficits in motor speed, coordination, visual memory, and visuospatial performance, which correspond to a developmental delay of up to 2 years (Harari et al., 2010). Recent research has also linked ADHD with organophosphates in children 8 to 15 years old (Bouchard, Bellinger, Wright, \& Weisskopf, 2010). This study assessed the urinary pesticide metabolite concentrations of nearly 1,200 school-aged children representative of the U.S. population. The authors found that children with higher levels of metabolite concentrations, particularly dimethyl alkylphosphate (DMAP), were more likely to meet the Diagnostic and Statistical Manual of Mental Disorders, 4th Edition (American Psychiatric Association, 1994), diagnostic criteria for ADHD. Notably, the level of pesticide exposure at which the authors observed this relationship is fairly common 
among U.S. children, suggesting a possible mechanism for the increasing incidence of ADHD nationwide (Bouchard et al., 2010). Furthermore, a recent study in the Netherlands reported that transplacental transfer of polybrominated flame retardants is associated with the poor neuropsychological development of school-aged children (Roze et al., 2009). Taken together, the literature suggests that levels of pesticide exposure that do not cause symptoms in pregnant mothers as well as levels of exposure common among the U.S. population can lead to neurological and behavioral deficits (e.g., motor, memory, and attention) in schoolaged children.

\section{Mechanism of Effect}

Pesticides may affect young children's neurological development and behavior through several pathways. Many classes of pesticides persist in the environment and body over time, such as DDT (Gilden et al., 2010). DDT has been used worldwide as an insecticide, with particular success in fighting malaria, but because of its persistence in the body it can lead to in utero and breastfeeding exposure in children (Ribas-Fito et al., 2006). Many chemical pesticides, including organophosphates, specifically work by targeting enzymes that regulate neurotransmitters, such as acetylcholine, which is released by motor neurons (Gilden et al., 2010). Because children may be exposed to numerous pesticides at various stages of life, it is difficult to assess the neurodevelopmental effects of specific pesticides (Colborn, 2006). With rodent studies, researchers have demonstrated that the developing brain is sensitive to cholinesterase inhibition at levels that would not be toxic to adults (Colborn, 2006) and that levels necessary for neurological effects are lower than those required to cause systemic toxicity (Slotkin, Levin, \& Seidler, 2006). Age and genetics may also play a role in how pesticide exposure affects individuals (Eskenazi et al., 2008).

\section{State of the Science and Research Implications}

Overall, research points to deleterious effects of pesticides on neurobehavioral development in young children. Although studies of children 2 years and younger report poorer outcomes with higher levels of exposure, psychomotor or mental development is inconsistently affected (Eskenazi et al., 2007; Torres-Sanchez et al., 2007). Various pesticide compounds have been linked to poor neurobehavioral outcomes, including verbal, memory, responsespeed, and possibly autism in preschoolaged children (Ribas-Fito et al., 2006; Roberts et al., 2007; Rohlman et al., 2005). Notably, school-aged children exposed to higher levels of pesticides have greater risk of ADHD (Xu et al., 2011). However, gaps persist in the literature regarding links among autism, psychomotor and mental development, and pesticides' mechanism of action. Many studies are limited by small sample sizes, emphasizing the need for future research to use pooled studies with similar methods and outcome measures. Additionally, accurately capturing all possible pesticide exposures in utero or postnatally remains a challenge. Animal studies will continue to inform this field of research because experimental studies are not ethically plausible.

Future research should include longitudinal, prospective, and larger studies of children's health and pesticide exposure risks. Additional work should be done to evaluate mechanisms of action and clarify some of the less consistent findings. Research must be ongoing to

Workplace Health Saf. Author manuscript; available in PMC 2014 November 28. 
determine the specific levels of chemical pesticides detrimental to children. A new federally funded study shows promise for providing additional information on children's health and the environment. The National Children's Study, led by several federal agencies, will follow 100,000 U.S. children prenatally to age 21 years to assess a variety of potential environmental influences on their health and development and should add insight into the effects of pesticides (Sattler \& Davis del, 2008).

\section{Implications}

Given the evidence about the potential links between prenatal and early childhood pesticide exposure and neurological and behavioral deficits, a variety of potential interventions should be tested at the policy, public health, and individual levels. It is vital to assess how children are exposed, the clinical implications of exposure, and education to improve understanding about exposure and outcomes. Significant areas include clinical practice and risk assessment, education, policy, and regulation.

\section{Practice}

Clinicians, including occupational and environmental health nurses and other health care providers, have a significant role to play in mitigating pesticide exposure risk and long-term effects through prevention, recognition, management, risk assessment, and treatment of environmental diseases (Rogers, McCurdy, Slavin, Grubb, \& Roberts, 2009). The American Nurses Association (ANA) mandates that nurses use current data to identify the potential for harm from a substance (even without complete information) and use the Precautionary Principle, which asserts that without conclusive evidence it is better to err on the side of caution and assume a negative association, to guide prevention, policy, and clinical decision making (Gilden et al., 2010). Women's health, neonatal, pediatric, community health, and occupational health nurses support families and women preparing for pregnancy by disseminating information about environmental hazards and chemical toxicants (Chalupka \& Chalupka, 2010; Sattler \& Davis del, 2008). Nurses are trusted professionals who educate clients effectively but may be limited in time. This situation may require women to seek information on their own, which may be an unreasonable expectation (Grason \& Misra, 2009). Little research has investigated what mothers and pregnant women know about environmental health threats (Grason \& Misra, 2009), indicating both a research and an educational need.

\section{Risk Assessment}

An especially vital task for occupational health nurses is assessing risk. Basic questions can be included in health histories (e.g., those recommended by the American College of Obstetrics and Gynecology [ACOG]) or through more detailed assessments (e.g., the Environmental and Occupational Health History Profile), providing a thorough understanding of potential home, community, and workplace pesticide exposures (Chalupka $\&$ Chalupka, 2010). Five strategies are necessary to create a child-protective approach to risk assessment: improved exposure assessments, enhanced toxicity testing, new toxicology models specific to children, a mechanistically based approach to hazard evaluation, and use 
of uncertainty and safety factors specific to children and their unique risks (Landrigan et al., 2004).

To understand exposure implications, identifying the etiology of possible exposure is crucial. Children can be exposed at home, via their parents' employment (e.g., parents are agricultural workers), in the community, or at school and day care (Sattler \& Davis del, 2008; Tulve et al., 2006). For instance, children living in agricultural areas have higher levels of urinary metabolites compared to urban children (Lambert et al., 2005). Recent research suggests that children have higher levels of pyrethroid insecticide metabolites in their systems than adults (Barr et al., 2010). A major route of pesticide exposure in young children is dietary ingestion. Organic diets have been popularized in the media and among the public, and several studies support their benefit in limiting pesticide exposure (Curl, Fenske, \& Elgethum, 2003; Lu, Barr, Pearson, Bartell, \& Bravo, 2006). However, residential pesticide use likely represents a greater risk and the risk changes with age as children engage more with their environments (Lu et al., 2006). School nurses in particular can play an important role in assessing schools and assisting parents in assessing homes for potential pesticide exposure risks.

\section{Education}

Education is critical to mitigating pesticide risk, both through anticipatory guidance for families and broader campaigns targeting the general public. Education should include information about potential exposures, alternatives to chemical pesticides, signs of intoxication, and risks of exposure. Parents should be counseled about potential risks of pesticides accumulating on their clothes, shoes, or skin if they live or work in agricultural areas (Chalupka \& Chalupka, 2010; Harrison, Partelow, \& Grason, 2009). Although workers may receive on-thejob training about pesticide safety, language or literacy barriers may necessitate provider counseling (Lucas \& Allen, 2009), such as advising farmworkers to prevent cross-contamination by showering and changing before returning home or holding infants (Lucas \& Allen, 2009). Ideally, education about potential pesticide exposures should begin prior to pregnancy; however, pregnancies are sometimes unplanned, so protective measures and educational campaigns should already exist in both the workplace and the home (Chalupka \& Chalupka, 2010). Women should avoid applying pesticides both before conception and during pregnancy, and occupational health nurses can assist workers in identifying potentially hazardous exposures at work and at home by conducting risk assessments (Chalupka \& Chalupka, 2010). Although chemical pesticides are frequently used in homes, schools, and workplaces, especially insecticides, non-chemical alternatives, when used appropriately, can negate the need for toxicants such as organophosphates.

Integrated pest management (IPM) is a useful framework that suggests setting an acceptable level for each pest before triggering action, monitoring and identifying pests, preventing pests' access to food, water, or shelter, and controlling pests with the least toxic option first (Gilden et al., 2010). Although specific definitions may vary, the leading principle of IPM is to use the least toxic pesticides after alternative methods have failed to prevent, mitigate, or eliminate pest infestations (Taylor \& Esdaille, 2010). One controversy with IPM is determining a consistent understanding of "least toxic" (Taylor \& Esdaille, 2010). Some 
research suggests that IPM might be more effective than traditional methods of pesticide application, but better cleaning and sanitation, more training and education, and better communication are needed to implement IPM (Taylor \& Esdaille, 2010).

An additional area for education is including environmental health content in medical and nursing curricula. Providers should conduct environmental health histories and risk assessments as well as educate clients and their families (Grason \& Misra, 2009). The National Environmental Education Foundation's Children's Environmental Health Faculty Champions Initiative has demonstrated preliminary success in building environmental health capacity among providers with train-the-trainer workshops for faculty members to better integrate environmental health into curricula (Rogers et al., 2009). This initiative could be replicated in additional schools of medicine and nursing to improve environmental health knowledge among providers.

Several articles and governmental sources have produced valuable compilations of resources providing education on environmental toxicants including pesticides (Chalupka \& Chalupka, 2010; Grason \& Misra, 2009). The national network of Pediatric Environmental Health Specialty Units (PEHSUs) is a useful resource to educate families by hosting experts across a variety of disciplines to provide information and counseling about environmental hazards (Chalupka \& Chalupka, 2010); however, they have not become a major data source on environmental health for schools (Paulson \& Barnett, 2010). The EPA's Integrated Risk Information System database, available online, provides information for those without toxicology training. The EPA database includes qualitative and quantitative information about environmental toxicants to underpin decision making, risk assessments, and regulatory mandates.

\section{Policy and Regulatory}

A life course approach can ensure that all aspects of a child's and a family's context is considered in developing policy initiatives (Grason \& Misra, 2009). Challenges to appropriate policy management of pesticide risks include the rapid development of new chemicals and increasing frequency of mixed pesticides use, along with insufficient safety evaluation of the majority of these products, especially for children (Landrigan et al., 2004). Policy strategies to mitigate environmental toxicants include public notification, mass media, product labeling, legislation and regulation, and surveillance (Grason \& Misra, 2009). Product labeling assists consumers to understand the risks of pesticide exposure, but labeling benefits may be limited by lack of health literacy and other literacy issues (Grason \& Misra, 2009). Print, radio, and television can improve the knowledge of the general public but lack quality control; official public notification, such as government websites, may only be accessed by individuals seeking that information (Grason \& Misra, 2009). Enforcement and regulation of safe agricultural working and housing conditions can also protect children (Lucas \& Allen, 2009). As policy advocates, occupational health nurses should encourage the use of IPM in their places of work and support funding for research (Gilden et al., 2010). Policies should be enacted at various governmental and institutional levels to encourage IPM, improved health care provider education regarding pesticides, appropriate regulation of pesticides, and research funding for pesticide safety studies. 
The government and its agencies can regulate the use of certain substances, which can have policy ramifications potentially benefiting children's health. The EPA is tasked with regulating pesticides in the United States, primarily under two statutes stipulating that the government, acting through the EPA, requires labeling and registration of pesticides to protect health, and setting maximum tolerable pesticide residues in food (EPA, 2010b). The Food and Drug Administration and the U.S. Department of Agriculture enforce these tolerances. The 1996 Food Quality Protection Act amended previous legislation to create additional regulations stipulating special protections for children and infants, establishing a single standard pesticide level across food types, providing incentives to develop crop protection for American farmers, and requiring regular re-evaluation of tolerance levels based on recent research data (EPA, 2010b). Researchers have also proposed the need for a new approach to determine the safety of pesticides. One recent study of how pesticide safety is determined and the potential negative neurodevelopmental effects critiqued the wide range of doses used in research; these doses should be more reflective of realistic exposure levels, acknowledge the need to assess pesticide impact across two generations, and reiterate the need to take protective action in the face of scientific uncertainty (Colborn, 2006). Risk assessment models should take into account children's multiple simultaneous exposures to different pesticides and should be constructed to capture acute or long-term effects (Landrigan et al., 2004).

The United States has experienced mixed success with regulating or banning pesticides because some pesticides linger in the environment and continue to contribute to poor neurobehavioral outcomes. For instance, higher levels of DDT have been linked to poor outcomes even after use of the pesticide had been limited for decades (Ribas-Fito et al., 2006). Evaluation of the effectiveness of the EPA's phase out of two types of organophosphates for residential use found that, over time, children's levels of exposure were significantly reduced and below published reference values, indicating restriction success (Wilson, Strauss, Iroz-Elardo, \& Chuang, 2010).

An additional area for policy and regulatory action is schools. Children spend a significant proportion of their time in school, and educational outreach also offers the opportunity to protect unborn children of pregnant women employed in or attending school. Although schools represent an area for potential pesticide exposure and place for educating parents about pesticides, few mechanisms are currently in place for protecting children's health at school. No federal or state agency is dedicated to verifying schools' environmental health policies or quality, and no systematic exposure data are collected at school (Paulson \& Barnett, 2010). A recent Centers for Disease Control and Prevention survey found that only one fourth of state education officials require districts to have an IPM plan, but recently, more than half of all states have established legislation restricting pesticide use in or around schools (Paulson \& Barnett, 2010). Implementing policies governing schools' environmental health and safety is complex, given the differing roles of federal, state, and local district management (Paulson \& Barnett, 2010) and the lack of policy standardization (Taylor \& Esdaille, 2010). Many of the existing policies protect adults working in schools but not explicitly children attending school; this situation should be addressed at the federal level (Paulson \& Barnett, 2010). A report specifying a plan to achieve IPM in all schools by 2015 was recently compiled by universities, government agencies, organized labor, and advocacy 
organizations (Taylor \& Esdaille, 2010). Additional recommendations include establishing effective standardized data collection, a federal strategy for states to improve schools' design and construction, additional funding for PEHSUs to address school health including inspections of schools and day care facilities, and required use of EPA screening tools (Paulson \& Barnett, 2010). Because IPM policies differ and may be insufficient to protect vulnerable children, federal legislation should standardize IPM in schools and effectively diminish exposure risk to both pesticides and pests. Legislation proposed in 2009 has not yet passed and will require the support of parents and educators (Taylor \& Esdaille, 2010).

A variety of clinical, educational, and policy recommendations may reduce the burden of pesticides' effects on children's neurologic and behavioral health. Occupational health nurses should:

- Use more thorough risk assessment tools (e.g., the Environmental and Occupational Health History Profile).

- Encourage organic diets over conventional diets for young children as affordable.

- Educate parents working in agriculture about the risks of bringing home pesticide residue and encourage their removing clothing and shoes and showering.

- Discourage women from spraying pesticides during pregnancy.

- Implement IPM in health care settings, homes, and schools using the least harmful substances; providers, parents, and maintenance staff should be educated about IPM.

- Educate health science students about environmental risks, including pesticides.

- Promote the use of IPM in schools via federal regulation.

- Use the Precautionary Principle and adopt a protective approach to tolerance levels set by federal regulatory agencies (e.g., EPA).

- Encourage policies to support IPM, improved environmental education for health care providers, appropriate regulation of pesticides, and research funding for pesticide safety studies among others.

\section{Conclusion}

Children are vulnerable to environmental toxicants, including pesticides, and therefore require special research and policy attention. In general, pesticides are associated with poor behavioral and neurological outcomes in young children, possibly due to their effect on neurotransmitters. Occupational health nurses and other health care providers can appropriately assess risk, conduct research, provide education, and support policies to address the potential impact of pesticides on children and adults.

\section{Acknowledgments}

This work was supported by grants K01-ES015 877 and R01-ES018858 from the National Institutes of Health and the National Institute of Environmental Health Sciences.

Workplace Health Saf. Author manuscript; available in PMC 2014 November 28. 


\section{References}

American Psychiatric Association. Diagnostic and statistical manual of mental disorders. 4th ed.. Arlington, VA: Author; 1994.

Barr DB, Olsson AO, Wong LY, Udunka S, Baker SE, Whitehead RD, et al. Urinary concentrations of metabolites of pyrethroid insecticides in the general U.S. population: National Health and Nutrition Examination Survey 1999-2002. Environmental Health Perspectives. 2010; 118(6):742-748. [PubMed: 20129874]

Bouchard MF, Bellinger DC, Wright RO, Weisskopf MG. Attention-deficit/hyperactivity disorder and urinary metabolites of organophosphate pesticides. Pediatrics. 2010; 125(6):e1270-e1277. [PubMed: 20478945]

Chalupka S, Chalupka AN. The impact of environmental and occupational exposures on reproductive health. Journal of Obstetric, Gynecologic, and Neonatal Nursing. 2010; 39(1):84-100.

Colborn T. A case for revisiting the safety of pesticides: A closer look at neurodevelopment. Environmental Health Perspectives. 2006; 114(1):10-17. [PubMed: 16393651]

Curl CL, Fenske RA, Elgethum K. Organophosphorous pesticide exposure of urban and suburban preschool children with organic and conventional diets. Environmental Health Perspectives. 2003; 111(3):377-382. [PubMed: 12611667]

Ecobichon DJ. Pesticide use in developing countries. Toxicology. 2001; 160(1-3):27-33. [PubMed: 11246121]

Engel SM, Berkowitz GS, Barr DB, Teitelbaum SL, Siskind J, Meisel SJ, et al. Prenatal organophosphate metabolite and organochlorine levels and performance on the Brazelton Neonatal Behavioral Assessment Scale in a multiethnic pregnancy cohort. American Journal of Epidemiology. 2007; 165(12):1397-1404. [PubMed: 17406008]

Eskenazi B, Marks AR, Bradman A, Harley K, Barr DB, Johnson C, et al. Organophosphate pesticide exposure and neurodevelopment in young Mexican-American children. Environmental Health Perspectives. 2007; 115(5):2911-2917.

Eskenazi B, Rosas LG, Marks AR, Bradman A, Harley K, Holland N, et al. Pesticide toxicity and the developing brain. Basic \& Clinical Pharmacology \& Toxicology. 2008; 102(2):1953-1958.

Gilden RC, Huffling K, Sattler B. Pesticides and health risks. Journal of Obstetric, Gynecologic, and Neonatal Nursing. 2010; 39(1):103-110.

Grason HA, Misra DP. Reducing exposure to environmental toxicants before birth: Moving from risk perception to risk reduction. Public Health Reports. 2009; 124(5):629-641. [PubMed: 19753941]

Harari R, Julvez J, Katsuyuki M, Barr D, Bellinger DC, Debes F, et al. Neurobehavioral deficits and increased blood pressure in school-age children prenatally exposed to pesticides. Environmental Health Perspectives. 2010; 118(6):890-896. [PubMed: 20185383]

Harrison, E.; Partelow, J.; Grason, H. Environmental toxicants and maternal and child health: An emerging public health challenge. Baltimore, MD: Johns Hopkins Bloomberg School of Public Health; 2009.

Horton MK, Rundle A, Camann DE, Boyd Barr D, Rauh VA, Whyatt RM. Impact of prenatal exposure to piperonyl butoxide and permethrin on 36-month neurodevelopment. Pediatrics. 2011; 127(3):e699-e706. [PubMed: 21300677]

Kofman O, Berger A, Massarwa A, Friedman A, Jaffar AA. Motor inhibition and learning impairments in schoolaged children following exposure to organophosphate pesticides in infancy. Pediatric Research. 2006; 60(1):88-92. [PubMed: 16788088]

Lambert WE, Lasarev M, Muniz J, Scherer J, Rothlein J, Santana J, et al. Variation in organophosphate pesticide metabolites in urine of children living in agricultural communities. Environmental Health Perspectives. 2005; 113(4):504-508. [PubMed: 15811843]

Landrigan PJ. Tighter regulation to protect the nation's children. Health Affairs. 2011; 30(5):851.

Landrigan PJ, Goldman LR. Protecting children from pesticides and other toxic chemicals. Journal of Exposure Science \& Environmental Epidemiology. 2011; 21(2):119-120. [PubMed: 21224896]

Landrigan PJ, Kimmel CA, Correa A, Eskenazi B. Children's health and the environment: Public health issues and challenges for risk assessment. Environmental Health Perspectives. 2004; 112(2):257-265. [PubMed: 14754581] 
Lu C, Barr DB, Pearson M, Bartell S, Bravo R. A longitudinal approach to assessing urban and suburban children's exposure to pyrethroid pesticides. Environmental Health Perspectives. 2006; 114(9):1419-1423. [PubMed: 16966099]

Lucas SF, Allen PJ. Reducing the risk of pesticide exposure among children of agricultural workers: How nurse practitioners can address pesticide safety in the primary care setting. Pediatric Nursing. 2009; 35(5):308-317. [PubMed: 19916348]

Paulson J, Barnett C. Who's in charge of children's environmental health at school? New Solutions. 2010; 20(1):3-23. [PubMed: 20359989]

Petit C, Chevrier C, Durand G, Monfort C, Rouget F, Garlantezec R, et al. Impact on fetal growth of prenatal exposure to pesticides due to agricultural activities: A prospective cohort study in Brittany, France. Environmental Health: A Global Access Science Source. 2010; 9

Quiros-Alcala L, Bradman A, Nishioka M, Harnly ME, Hubbard A, McKone TE, et al. Pesticides in house dust from urban and farmworker households in California: An observational measurement study. Environmental Health: A Global Access Science Source. 2011; 10:19. [PubMed: 21410986]

Rauh VA, Garfinkel R, Perera R, Andrews H, Barr D, Whitehead D, et al. Impact of prenatal chlorpyrifos exposure on neurodevelopment in the first three years of life among inner-city children. Epidemiology. 2006; 17(6):S102-S102.

Ribas-Fito N, Torrent M, Carrizo D, Munoz-Ortiz L, Julvez J, Grimalt JO, et al. In utero exposure to background concentrations of DDT and cognitive functioning among preschoolers. American Journal of Epidemiology. 2006; 164(10):955-962. [PubMed: 16968864]

Roberts EM, English PB, Grether JK, Windham GC, Somberg L, Wolff C. Maternal residence near agricultural pesticide applications and autism spectrum disorders among children in the California Central Valley. Environmental Health Perspectives. 2007; 115(10):1482-1489. [PubMed: 17938740]

Rogers B, McCurdy LE, Slavin K, Grubb K, Roberts JR. Children's Environmental Health Faculty Champions Initiative: A successful model for integrating environmental health into pediatric health care. Environmental Health Perspectives. 2009; 117(5):850-855. [PubMed: 19478972]

Rohlman DS, Arcury TA, Quandt SA, Lasarev M, Rothlein J, Travers R, et al. Neurobehavioral performance in preschool children from agricultural and non-agricultural communities in Oregon and North Carolina. Neurotoxicology. 2005; 26(4):589-598. [PubMed: 16112324]

Roze E, Meijer L, Bakker A, Van Braeckel KNJA, Sauer PJJ, Bos AF. Prenatal exposure to organohalogens, including brominated flame retardants, influences motor, cognitive, and behavioral performance at school age. Environmental Health Perspectives. 2009; 117(12):19531958. [PubMed: 20049217]

Sattler B, Davis del BA. Nurses'role in children' environmental health protection. Pediatric Nursing. 2008; 34(4):329-339. [PubMed: 18814568]

Slotkin TA, Levin ED, Seidler FJ. Comparative developmental neurotoxicity of organophosphate insecticides: Effects on brain development are separable from systemic toxicity. Environmental Health Perspectives. 2006; 114(5):746-751. [PubMed: 16675431]

Taylor AK, Esdaille K. Integrated pest management policies in America's schools: Is federal legislation needed? New Solutions. 2010; 20(1):73-80. [PubMed: 20359992]

Torres-Sanchez L, Rothenberg SJ, Schnaas L, Cebrian ME, Osorio E, Del Carmen Hernandez M, et al. In utero p,p'-DDE exposure and infant neurodevelopment: A perinatal cohort in Mexico. Environmental Health Perspectives. 2007; 115(3):435-439. [PubMed: 17431495]

Tulve NS, Jones PA, Nishioka MG, Fortmann RC, Croghan CW, Zhou JY, et al. Pesticide measurements from The First National Environmental Health Survey of Child Care Centers using a multi-residue GC/MS analysis method. Environmental Science \& Technology. 2006; 40(20): 6269-6274. [PubMed: 17120552]

U.S. Environmental Protection Agency. Pesticides. 2010a Retrieved from www.epa.gov/pesticides.

U.S. Environmental Protection Agency. The Food Quality Protection Act (FQPA) background. 2010b Retrieved from www.epa.gov/opp00001/regulating/laws/fqpa/backgrnd.htm.

Wilson NK, Strauss WJ, Iroz-Elardo N, Chuang JC. Exposures of preschool children to chlorpyrifos, diazinon, pentachlorophenol, and 2,4-dichlorophenoxyacetic acid over 3 years from 2003 to 2005: 
A longitudinal model. Journal of Exposure Science and Environmental Epidemiology. 2010; 20(6):546-558. [PubMed: 19724304]

Xu XH, Nembhard WN, Kan HD, Kearney G, Zhang ZJ, Talbott EO. Urinary trichlorophenol levels and increased risk of attention deficit hyperactivity disorder among US schoolaged children. Occupational and Environmental Medicine. 2011; 68(8):557-561. [PubMed: 21540483] 


\section{IN SUMMARY}

1. Widely used around the world, pesticides play an important role in protecting health, crops, and property. However, pesticides may also have detrimental effects on human health, with young children among the particularly vulnerable.

2. Recent research suggests that even low levels of pesticide exposure can affect young children's neurological and behavioral development. Evidence shows a link between pesticides and neonatal reflexes, psychomotor and mental development, and attention-deficit hyperactivity disorder.

3. Implications include a need for improved risk assessment and health histories by clinicians, greater education at all levels, more common use of integrated pest management, and continued policy and regulatory strategies to mitigate the effects of and the need for pesticides. 